\title{
Visual outcomes in children in Malawi following retinopathy of severe malaria
}

\author{
N A V Beare, C Southern, K Kayira, T E Taylor, S P Harding
}

Br J Ophthalmol 2004;88:321-324. doi: 10.1136/bjo.2003.025924

Series editors: W V Good, S Ruit

See end of article for authors' affiliations

......................

Correspondence to: Nicholas Beare, FRCOphth, St Paul's Eye Unit, Royal Liverpool University Hospital, Prescot Street, Liverpool L7 8XP,

UK; nbeare@

btinternet.com

Accepted for publication 11 August 2003

\begin{abstract}
Aim: To investigate whether retinal changes in children with severe malaria affect visual acuity 1 month after systemic recovery.

Methods: All children with severe malaria admitted to a research ward in Malawi during one malaria season were examined by direct and indirect ophthalmoscopy. Visual acuity was tested in those attending follow up by Cardiff cards, Sheridan-Gardiner single letters, or Snellen chart.

Results: 96 (68\%) children attended follow up, of whom 83 (86\%) had visual acuity measured. Cardiff cards were used in 47 (57\%) children, and Sheridan-Gardiner letters or Snellen chart in 29 (35\%). There was no significant difference in the mean logMAR visual acuity between groups with or without macular whitening $(0.14$ versus $0.16, p=0.55)$. There was no trend for worse visual acuity with increasing severity of macular whitening $(p=0.52)$ including patients in whom the fovea was involved $(p=0.32)$. Six $(4.2 \%)$ children had cortical blindness after cerebral malaria, and all six had other neurological sequelae. Ophthalmoscopy during the acute illness revealed no abnormalities in four of these children.

Conclusion: Retinal changes in severe malaria, in particular macular whitening, do not appear to affect visual acuity at 1 month. This supports the hypothesis that retinal whitening is due to reversible intracellular oedema in response to relative hypoxia, caused by sequestered erythrocytes infected by Plasmodium falciparum. Impaired visual functioning after cerebral malaria is not attributable to retinal changes and appears to be a cortical phenomenon.
\end{abstract}

$\mathrm{T}$ he visual outcome of children after severe malaria has not previously been systematically investigated. The retinal signs associated with severe malaria in children in Africa are well documented ${ }^{1-3}$ and include opacification or whitening of the macula and fovea (fig 1), vessel changes, multiple retinal haemorrhages, and papilloedema. The pathophysiology of these signs has not been fully elucidated.

Despite these retinal changes, which are sometimes marked and widespread, severe malaria is not documented as a cause of poor vision in African children. ${ }^{4}$ Anecdotally isolated visual loss does not appear to be a consequence of severe or cerebral malaria.

Cortical blindness is a well recognised neurological sequela of cerebral malaria, ${ }^{5-8}$ and is often accompanied by other neurological deficits. Cortical blindness is usually a presumptive diagnosis, without assessment by an ophthalmologist. Most cortical blindness caused by cerebral malaria resolves over a matter of weeks. ${ }^{5}$

Our study aimed to investigate whether retinal changes, particularly macular whitening, affected visual acuity in children recovering from severe malaria.

\section{METHODS}

The study population included all children with severe malaria admitted to the Malaria Research Project ward, Department of Paediatrics, Queen Elizabeth Central Hospital, Blantyre, Malawi during a single malaria season. Most subjects had cerebral malaria defined as an acute onset of coma, scoring 2 or less on the Blantyre coma scale for at least 4 hours after admission, in the presence of $P$ falciparum parasitaemia. ${ }^{9}$ Other explanations for altered consciousness such as meningitis, hypoglycaemia, or post-ictal state were excluded. Other children had severe malarial anaemia defined by a haematocrit below 15\% but without deep coma, or severe malaria without other defining features. All children were treated according to a protocol with intravenous quinine and fluids. Anticonvulsants, glucose, and antipyretics were given according to need.

All children were examined by direct and indirect ophthalmoscopy after pupil dilatation with tropicamide $1 \%$ and phenylephrine $2.5 \%$, and the retinal findings recorded and graded for severity on standardised charts. ${ }^{10}$ Macular whitening is generally centred on the fovea, with sparing of the foveola, and the extent to which the fovea was involved was graded separately as foveal whitening. Ophthalmoscopy was performed on admission and daily thereafter subject to the depth of coma and cooperation. The eye findings used in the analysis were the most advanced for the child, regardless of when these changes were documented during admission.

Measurement of visual acuity was attempted on patients who survived and attended for hospital follow up, which was usually arranged for 4 weeks after discharge. No patients wore glasses and visual acuity was tested unaided in all cases. The vision was measured by an ophthalmologist with a Malawian nurse to communicate with children and parents in their language.

Different methods were used to measure visual acuity depending on the age, understanding, and cooperation of the child. The methods used were Cardiff preferential looking cards, Sheridan-Gardiner single letters with matching chart, Snellen chart with matching chart, and Snellen chart alone. Lea symbols were also trialled but their use was discontinued.

The right eye was used for analysis except when macular whitening was worse on the left; or when vision was only available with both eyes open, in which case the least affected eye was used. The visual acuities were converted to $\log$ MAR acuities for analysis. The difference between the mean $\log$ MAR acuities were compared by a Student's $t$ test. Trend in visual outcome according to severity of macular whitening was analysed by Cuzick's non-parametric test for trend. 


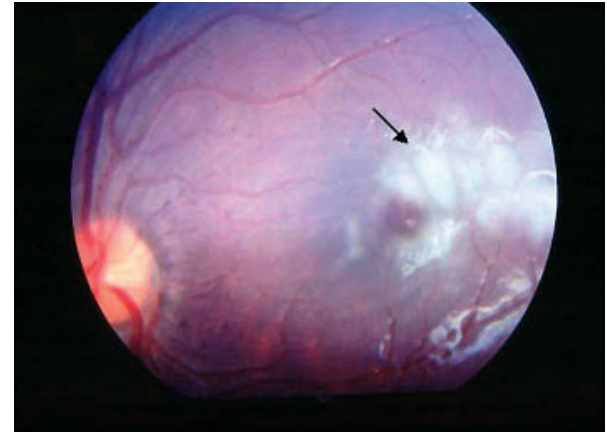

Figure 1 Severe macular whitening (arrowed), involving temporal fovea but sparing foveola, in Malawian child with cerebral malaria.

\section{RESULTS}

In all, 162 children were examined by funduscopy during the study period, and 20 (12\%) died; 143 had cerebral malaria, seven had severe anaemia, and 12 had severe malaria alone. Ninety six (68\%) survivors attended for follow up, of whom $83(86 \%)$ were sufficiently cooperative for visual acuity testing, and form the basis for the remaining analysis.

The median age was 3 years and 4 months (interquartile range 22-60 months), and there were $44(53.0 \%)$ females. Seventy nine of the patients had cerebral malaria, three had severe malarial anaemia and one had severe malaria alone. Fundus signs of severe malaria were present in 38 (46\%) while in hospital, including 31 (37\%) with macular whitening.

\section{Visual acuity at follow up}

The visual acuity was measured at a median of 27 days (interquartile range 22-29 days) after discharge. Cardiff cards were used for testing 47 (57\%) children, SheridanGardiner single letters for $18(22 \%)$, Snellen chart for 11 (13\%), including seven $(8 \%)$ using a matching card, and the method used was not recorded for two children. Lea symbols were trialled on a number of patients, and were the only record of vision for one. Four children with persistent neurological sequelae could only be assessed according to their ability to fix and follow.

The measured visual acuities for different degrees of macular whitening are shown in figure 2. The difference in the mean $\log$ MAR acuities between children who had had macular whitening (0.14) and those who had not (0.16) was not significant $(\mathrm{p}=0.55)$. Similar results were obtained for children with foveal whitening $(p=0.70)$. There were similar proportions with visual acuity of better than 6/12 in groups with and without macular whitening $\left(\chi^{2}, \mathrm{p}=0.60\right)$.

There was no trend of worsening visual acuity with increasing severity of macular or foveal whitening $(p=0.52$ and $p=0.32$ respectively). Figure 3 shows the maculae of two patients with moderate macular whitening whose acuity was recorded as 6/12 and 6/9 4 weeks after discharge.

Peripheral whitening, vessel changes, and capillary whitening were not associated with poor visual acuity at follow up. Four $(4.8 \%)$ patients had papilloedema, none had neurological sequelae, and all had a visual acuity of $6 / 6$ or $6 / 7.5$ recorded at follow up.

\section{Neurological sequelae and cortical blindness}

Of the 143 patients with cerebral malaria who had ophthalmoscopy during the recruitment period, $10(7.0 \%)$ were discharged with neurological sequelae, including six $(4.2 \%)$ with apparent cortical blindness. None could fix or follow at discharge and all had other neurological deficits (table 1). Four of the six with cortical blindness had normal

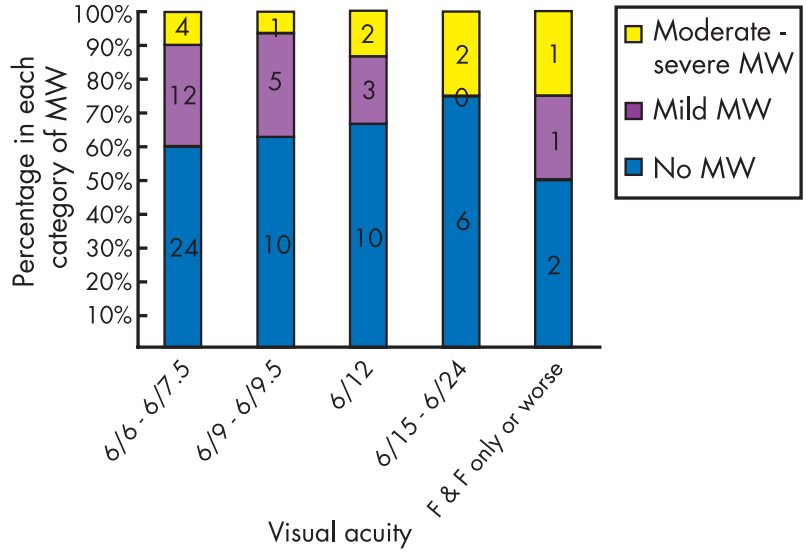

Figure 2 Percentage of patients with no, mild, or moderate to severe macular whitening (MW) with each level of visual acuity at 1 month follow up. Numbers of patients indicated. $F \& F=$ fixing and following.

funduscopic examinations while in hospital; one had mild macular whitening, and one had severe retinopathy, including severe macular whitening.

One patient had congenital cataracts and pre-existing developmental delay, and was excluded from the analysis of visual outcomes. One patient, with normal fundi in hospital, made a neurological and visual recovery within 2 weeks. Two others improved during follow up, and were able to fix and follow targets.

\section{DISCUSSION}

Our study did not identify any loss of visual acuity attributable to retinal changes in children identified during their acute episode of malaria. Of the retinal signs in severe malaria, macular whitening might be expected to cause visual deficit as it is centred on, and often involves, the fovea. Other distinct features of retinopathy in malaria mostly affect the peripheral fundus. Macular whitening is a patchy opacification and thickening of the inner retinal layers, with sparing the foveola ${ }^{2}$ (fig 1), which we found in 37\% of children with severe malaria. However, there was no difference in the level of vision between patients who had had macular whitening, including foveal involvement, and those who had not. This has implications for theories of pathogenesis of retinal signs in cerebral malaria.

It was not possible to measure visual acuity during the acute illness, because children were either in coma or prostrate. Patients were discharged soon after their coma and parasitaemia resolved, hence an accurate measure of visual acuity on discharge would not have been possible. The proportion of children attending for hospital follow up (68\%) was high for this setting, and we were able to record acuity in $86 \%$.

The methods used to measure visual acuity in young children differ according to the subject's age, language development, and cooperation. Tests that involve preferential looking at graduated targets of interest, such as Cardiff cards, are used with preverbal children. Verbal children can identify pictures or symbols, or match optotypes or letters to ones presented at a distance. Sheridan-Gardiner single letters are commonly used for this, and once subjects know letters, they can read the single optotypes before graduating to a Snellen chart.

We encountered a number of difficulties in measuring vision in young children with tasks that were often outside their cultural norms. The measurement of visual acuity was done in a hospital environment during a follow up visit 


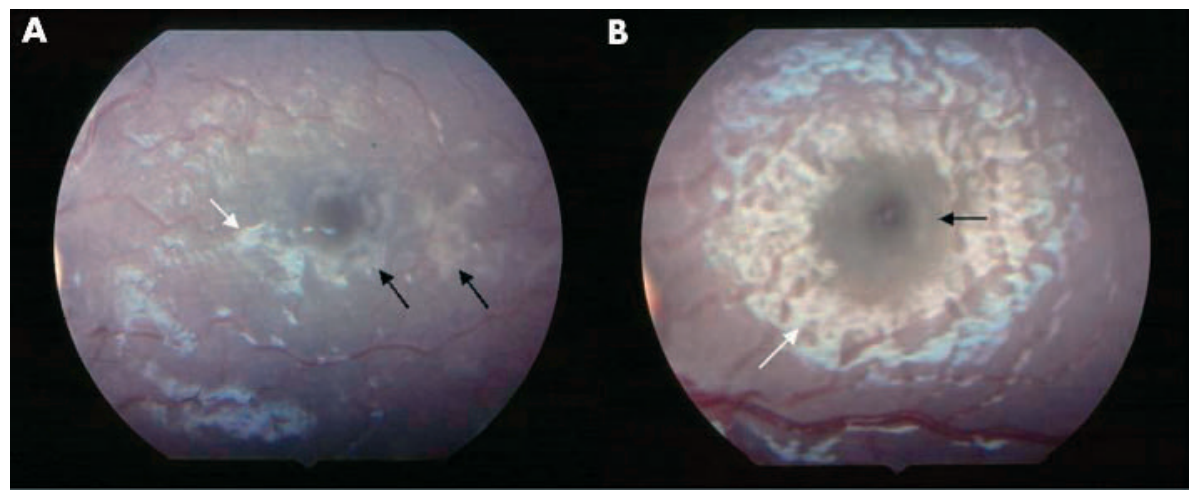

Figure 3 Moderate macular whitening circumferential to foveola (black arrows) in two children with cerebral malaria. The vision 1 month after systemic recovery was (A) $6 / 12$ and (B) $6 / 9$ (white arrows show artefact).

where capillary blood was routinely sampled, and this may have reduced cooperation.

Lea symbols, symbolic optotypes, which all blur to a circle, have been developed for children. It has been suggested they eliminate cultural biases ${ }^{11}$ and have been used successfully with native American children. ${ }^{12}$ We found verbal Malawian children were less good at identifying Lea symbols than matching letters. This may be because children in Malawi, particularly ones from rural areas, are not familiar with abstract pictures or symbols. They generally do not have access to picture books or drawing materials. They do have some exposure to letter shapes, but do not have systematic preschool teaching.

Cardiff preferential looking cards were used for children from 6 months, up to 5 years. Visual acuity in the verbal children was first attempted using Sheridan-Gardiner letters and a matching card, and if they were unable to match letters then Cardiff cards were used. The unfamiliarity with letters and lack of schooling may account for the necessity of using Cardiff cards in relatively older children. These children were also not sufficiently cooperative to perform acuity testing with the illiterate E chart.

Sheridan-Gardiner letters with a matching card were used for children aged 4-6 $1 / 2$ years. Children from 6 up to 14 years were generally able to use the Snellen chart with or without a matching card. Refraction was not done, which may have reduced some visual acuities recorded, but this would have been a consistent effect across groups compared.

The necessity of using different methods of vision testing in different age groups introduces a potential age bias. However, there was not any difference in the age of children with and without macular whitening (Student's $t$ test, $p=0.70$ ). We therefore do not believe that this has introduced systematic bias into our analysis.

During the course of this study there were six $(4.2 \%)$ patients with cortical blindness after cerebral malaria, who did not fix and follow large objects on discharge. This incidence is consistent with those reported in studies of neurological sequelae after cerebral malaria in children (3.6$5.9 \%) .{ }^{568}$ These studies did not include an ophthalmologist's assessment, and results of dilated funduscopy were not reported.

One study of cortical blindness in a tertiary ophthalmic clinic in Nigeria found eight of 22 (36\%) cases were caused by cerebral malaria. All cases had normal fundi, most (6/8) had other associated neurological deficits, and seven had some visual recovery within 6 weeks. ${ }^{13}$ All cases of cortical blindness reported here were accompanied by other neurological deficits, most commonly aphasia, deafness, ataxia, and hypotonia.

Four of the patients who were unable to fix and follow, had normal ocular fundi during admission. We infer that lack of visual behaviour acutely after cerebral malaria is not due to retinopathy, and that it is usually associated with other sensory or motor deficits. It indeed appears most likely to be a cortical phenomenon. Retinal changes including macular whitening, do not appear to contribute to apparent visual deficits following cerebral malaria.

Cortical blindness does not appear to be related simply to raised intracranial pressure indicated by papilloedema during the acute illness. The four patients in this group with papilloedema during admission all had good acuity on recovery.

Macular whitening does not closely resemble exudation, or extracellular oedema, and the results of this study support a different pathogenesis. Macular oedema centred on the fovea would be expected to affect children's visual acuity, and would normally take longer than a month to resolve.

On fluorescein angiography macular whitening is not accompanied by capillary non-perfusion or fluorescein leakage. ${ }^{1}$ We postulate that macular whitening is caused by cellular swelling of the inner retinal layers due to relative

Table 1 Six patients with cortical blindness following cerebral malaria. All patients did not fix or follow at time of discharge

\begin{tabular}{|c|c|c|c|c|}
\hline $\begin{array}{l}\text { Age } \\
\text { (months) }\end{array}$ & Retinal changes & Other deficits on discharge & Day of follow up & Vision at follow up \\
\hline 23 & Normal & $\begin{array}{l}\text { Aphasic, deaf, hypotonic, } \\
\text { hemiparesis }\end{array}$ & 39 & No $F \& F$ \\
\hline 36 & Normal & Aphasic, deaf & 14 & $6 / 9.5$ \\
\hline 22 & Mild & Ataxic & 30 & $F$ \& F only \\
\hline 26 & Normal & Aphasic, ataxic, hypotonic* & 7 & No $F \& F$ \\
\hline 57 & Severe & Decorticate, deaf & 14 & No $F \& F$ \\
\hline 15 & Normal & Hemiparesis, aphasic, gaze palsy & & $F$ \& $F$ large objects \\
\hline
\end{tabular}

$F \& F=$ fixing and following

*Previous developmental delay and congenital cataracts and therefore excluded from analysis of outcomes. 
hypoxia. Macular whitening is similar in appearance and angiographic findings to patchy ischaemic retinal whitening recently reported to be an uncommon early feature of central retinal vein occlusion with good visual recovery. ${ }^{14}$

In cerebral malaria intravascular sequestration of erythrocytes parasitised by $P$ falciparum occurs in both the brain and the retina. ${ }^{15}$ The parasites metabolise oxygen and haemoglobin, which we believe causes a relative deoxygenation or hypoxia leading to intracellular oedema, and hence reversible opacification. Electroretinographic evidence suggests that ganglion cells are dysfunctional in macular whitening which would account for the typical sparing of the foveola. ${ }^{16}$ With resolution of the metabolic deficit intracellular oedema would reverse, allowing a normal visual acuity on recovery.

Other possible causes of intracellular swelling are toxic products produced by $P$ falciparum, such as glycosylphosphatidylinositol (GPI), ${ }^{17}{ }^{18}$ high concentrations of cytokines such as tumour necrosis factor $\alpha^{19}$ or local factors such as nitric oxide, all of which have been shown to be important in the pathogenesis of cerebral malaria. ${ }^{20}{ }^{21}$ However, macular whitening is centred on the most metabolically active part of the retina, without predilection for perivascular areas most exposed to these blood borne elements. We therefore believe that this makes a hypoxic explanation more likely. Whether this has relevance to cerebral pathogenesis remains speculative.

Other retinal changes, particularly retinal vessel whitening, are in the fundal periphery. Detailed visual field analysis of the young infants in our population would not be possible, but further work with older children with cerebral malaria would show whether these peripheral vessel changes have hitherto undetected effects on the visual field.

We conclude that retinal changes in severe malaria do not appear to affect the visual acuity at 1 month. This makes exudation an unlikely cause, and supports the theory that macular whitening is due to reversible cellular swelling in response to a metabolic deficit.

\section{ACKNOWLEDGEMENTS}

We thank Professor Broadhead and the staff of the Department of Paediatrics, Queen Elizabeth Central Hospital; and the staff of the Malaria Research Project, particularly sister Rose Mazengera.

The study was funded by the British Council for the Prevention of Blindness and the Beit Trust. Clinical care of patients was under a programme funded by the Wellcome Trust, and the US National Institutes of Health, grant number NIH RO11 AO34969.

\section{Authors' affiliations}

N A V Beare, C Southern, S P Harding, St Paul's Eye Unit, Royal Liverpool University Hospital, Liverpool, UK

K Kayira, T E Taylor, Malaria Project and Wellcome Trust Laboratories, College of Medicine, Blantyre, Malawi
T E Taylor, College of Osteopathic Medicine, Michigan State University, East Lansing, USA

\section{REFERENCES}

1 Hero M, Harding SP, Riva CE, et al. Photographic and angiographic characterization of the retina of Kenyan children with severe malaria. Arch Ophthalmol 1997; 115:997-1003.

2 Lewallen S, Harding SP, Ajewole J, et al. A review of the spectrum of clinical ocular fundus findings in P falciparum malaria in African children with a proposed classification and grading system. Trans R Soc Trop Med Hyg 1999;93:619-22.

3 Lewallen S, Bakker H, Taylor TE, et al. Retinal findings predictive of outcome in cerebral malaria. Trans R Soc Trop Med Hyg 1996;90:144-6.

4 Gilbert CE, Wood M, Waddel K, et al. Causes of childhood blindness in east Africa: results in 491 pupils attending 17 schools for the blind in Malawi, Kenya and Uganda. Ophthalmic Epidemiol 1995;2:77-84.

5 Bondi FS. The incidence and outcome of neurological abnormalities in childhood cerebral malaria: a long-term follow-up of 62 survivors. Trans $R$ Soc Trop Med Hyg 1992;86:17-9.

6 Carme B, Bouquety JC, Plassart H. Mortality and sequelae due to cerebral malaria in African children in Brazzaville, Congo. Am J Trop Med Hyg 1993:48:216-21.

7 Meremikwu MM, Asindi AA, Ezedinachi E. The pattern of neurological sequelae of childhood cerebral malaria among survivors in Calabar, Nigeria. Cent Afr J Med 1997;43:231-4.

8 Brewster DR, Kwiatkowski D, White NJ. Neurological sequelae of cerebral malaria in children. Lancet 1990;336:1039-43.

9 Molyneux ME, Taylor TE, Wirima JJ, et al. Clinical features and prognostic indicators in paediatric cerebral malaria: a study of 131 comatose Malawian children. Q J Med 1989:71:441-59.

10 Beare NA, Southern C, Lochhead J, et al. Inter-observer concordance in grading retinopathy in cerebral malaria. Ann Trop Med Parasitol 2002;96:105-8.

11 Repka MX. Use of Lea symbols in young children. Br J Ophthalmol 2002;86:489-90.

12 Miller JM, Dobson VM, Harvey EM, et al. Astigmatism and amblyopia among Native American Children (AANAC): design and methods. Ophthalmic Epidemiol 2000;7:187-207.

13 Olurin $\mathrm{O}$. The aetiology of cortical blindness in Nigeria. Afr J Med Sci 1970;1:357-67.

14 Browning DJ. Patchy ischemic retinal whitening in acute central retinal vein occlusion. Ophthalmology 2002;109:2154-9

15 Lewallen S, White VA, Whitten RO, et al. Clinical-histopathological correlation of the abnormal retinal vessels in cerebral malaria. Arch Ophthalmol 2000;118:924-8.

16 Harding SP, Lochhead J, Movaffaghy A, et al. Electroretinography of severe malaria in Malawian children. Invest Ophthalmol Vis Sci 1999;40:S148.

17 Schofield L, Novakovic S, Gerold P, et al. Glycosylphosphatidylinositol toxin of Plasmodium up-regulates intercellular adhesion molecule-1, vascular cell adhesion molecule-1, and E-selectin expression in vascular endothelial cells and increases leukocyte and parasite cytoadherence via tyrosine kinasedependent signal transduction. J Immunol 1996;156:1886-96.

18 Tachado SD, Gerold P, McConville MJ, et al. Glycosylphosphatidylinositol toxin of Plasmodium induces nitric oxide synthase expression in macrophages and vascular endothelial cells by a protein tyrosine kinase-dependent and protein kinase C-dependent signaling pathway. J Immunol 1996;156:1897-907

19 Grau GE, Taylor TE, Molyneux ME, et al. Tumor necrosis factor and disease severity in children with falciparum malaria. N Engl J Med 1989:320:1586-91.

20 Newton CR, Taylor TE, Whitten RO. Pathophysiology of fatal falciparum malaria in African children. Am J Trop Med Hyg 1998;58:673-83.

21 Malaguarnera L, Musumeci S. The immune response to Plasmodium falciparum malaria. Lancet Infect Dis 2002;2:472-8. 